\title{
Research on the International Carbon Emission Trading Mechanism
}

\author{
Ming Zeng ${ }^{1}$, Shiyu Meng ${ }^{1}$ a , Zhuhan Long ${ }^{1}$, Liyi Tian ${ }^{1}$, Chenjun Sun ${ }^{2}$ \\ ${ }^{1}$ School of Economics and Management, North China Electric Power University, Beijing 102206, \\ China; \\ ${ }^{2}$ State Grid Hebei Electric Power Company, Hebei 050000, China. \\ a799079198@qq.com
}

\begin{abstract}
Carbon emissions trading is an important part of international environmental cooperation. It is also a comparatively novel applied research field of environmental economics and institutional economics in international trade. The existing carbon trading mechanisms mainly have problems such as cost-efficiency asymmetry and differences of interests. In order to solve these problems, this paper proposes to construct a new emission trading mechanism. The new mechanism is based on the principle of common but differentiated responsibilities, cost-sharing principle and selective incentive principle. It focuses on the challenges and opportunities faced by the trading of emission rights based on transaction cost theory and the construction of a global carbon emissions trading mechanism.
\end{abstract}

Keywords: Climate change, Emissions trading, Carbon emissions trading.

\section{Introduction}

Greenhouse gases produced by human production and living have a major impact on the global environment. The rise of greenhouse gas emissions has caused rising global temperatures and anomalies in the climate. The threats that they pose include sea levels rising, extinction of endangered species, instability in food production, destruction of global ecosystems, etc. In today's international community, climate change has become an important agenda in addition to security and economic issues. Among the measures in response to climate change, reducing greenhouse gas emissions is currently the most effective method. The international community has been trying to find a cooperation mechanism to limit greenhouse gas emissions and to meet the challenges posed by climate change.

The UN Framework Convention on Climate Change issued in 1992 is the broadest consensus reached by the international community so far. The Convention set forth the ultimate goal of "stabilizing the concentration of greenhouse gases in the atmosphere at a level that prevents the climate system from being perceived as dangerous". In 2005, the "Kyoto Protocol" officially entered into force and entered the track of actual operation. Therefore, the right to greenhouse gas emissions has become a scarce resource and trading of emission rights has emerged.

Climate change not only affects everyone's life, affects the survival and development of a country, but also profoundly affects the international relations and international political structure in the world today. Negotiations between countries on climate change have been under way since the 1990s and have now entered the post-Kyoto era. The main agenda is the emission reduction commitments of developed and developing countries after 2012. The basic pattern of confrontation between the camps of developed and camps of developing countries in the north and the south is the most important feature of climate negotiations. As a representative of developing country, China uses the method of developed countries to achieve greenhouse gas emission reduction through market means for reference, realizes market transactions of emission rights among domestic enterprises, and controls greenhouse gas emissions to the maximum extent. In this way, China can establish the image of a responsible big country, and win the right to speak in international climate negotiations, and occupy an advantageous position in the post-Kyoto era of international climate politics. 


\section{Principles of International Carbon Trading Mechanism Construction}

\subsection{Common but Differentiated Responsibilities}

When carrying out international cooperation to prevent global warming, the responsibilities of developing and developed countries are different. They must distinguish their responsibilities rather than talk about common responsibilities in an abstract manner. The population of developed countries only accounts for $25 \%$ of the world's population, but it consumed and is consuming $80 \%$ of the world's resources. Since the 1860 s, the oil and coal that developed countries have burned during the industrialization process have increased the carbon dioxide content in the atmosphere by $60 \%$. Developed countries have accumulated more than 900 tons of carbon dioxide per capita since the industrial revolution. As the representative of developing countries, China has only 88 tons, and per capita emission represents climate responsibility. Therefore, international environmental cooperation must follow the principle of common but differentiated responsibilities.

\subsection{Principle of Cost Sharing}

The cost of international carbon emissions trading mechanisms is directly proportional to their scale, and the huge global cooperation costs constitute economic constraints and cost constraints for the collective actions of the mechanism. Therefore, in order to enable collective action to be carried out effectively, it is necessary to construct a cost-sharing mechanism for collective action of global carbon emission trading mechanisms. To this end, the international carbon emissions trading mechanism still adheres to the three extraterritorial emission reduction mechanisms, including the joint implementation mechanism, the clean development mechanism, and the emissions trading mechanism proposed by the Kyoto Protocol, to help countries that assume emission reduction responsibilities fulfill emission reduction targets.

The core of the extraterritorial emission reduction mechanism lies in the fact that the countries that bear the responsibility of reducing emissions can use these three mechanisms to obtain offsets for emission reductions outside of their own countries, thus achieving a significant reduction in the cost of abatement across the globe. Due to the different levels of economic development in various countries within the international carbon emissions trading mechanism, there is a wide gap between economic strength and emission reduction potential. Therefore, the global internal sharing agreement can adjust the emission reduction targets of various countries and rearrange them according to the energy sources and consumption conditions of various countries, thereby reduce the emission reduction costs of various countries, and effectively achieve the emission reduction targets on a monthly basis.

\subsection{Principle of Selective Incentives}

The international carbon emissions trading mechanism implements a strong selective incentive mechanism for members. Punishment of parties that have violated the regulations and failed to meet the emission reduction targets shall be stipulated in the provisions of the trading mechanism. In this way, the parties will be punished in the form of fines, etc. for failing to fulfill their emission reduction obligations. At the same time, publicizing the accomplishment of the emission reduction obligations of all participating parties will achieve incentives for these countries to reduce emissions. The international carbon trading mechanism conducts qualification examinations for participating countries, and can only join after review; after joining, they must not arbitrarily quit on the ground of damaging the interests of the country, and any exit will be punished. For the joining and withdrawing mechanism, we can draw lessons from the EU's temporary exit clause.

\section{Emission Trading Based on Transaction Cost Theory}

The policy design of international carbon emissions trading stems from emissions trading in environmental economic policies. In the policy options for governance of the environment, there is a game between the government and the enterprise. The government can control the company's 
pollution emissions through direct control, sewage charges, and emissions trading. Direct regulation means that the government directly limits the amount of pollutants discharged by enterprises according to the optimal amount of pollutants in the region, which reflects the government's management functions. The sewage charges refer to the government's taxation or implementation of charges on sewage companies. The essence of this is the introduction of a so-called Pigouvian tax and the use of administrative measures to internalize externalities. Emissions trading refers to the government's introduction of a market mechanism to allow polluters to trade pollutant discharge indicators based on determining the optimal amount of total emissions. This is actually a combination of government regulation and market mechanisms.

In recent decades, the deepening of industrialization and economic development have led to a high growth of energy and resource consumption on a global scale, and the consequent severe environmental pollution. How to effectively deal with environmental pollution has become an urgent issue that governments and international communities have to seriously face. Due to the negative externalities of pollutant emissions, many governments have long used administrative and legal measures to control pollution and protect the environment. Although they have achieved certain results, they have also caused some problems. Because administrative measures to control pollution are almost impossible to achieve a balance between costs and benefits, in the current situation where environmental protection costs are getting higher and higher, the governance of pollution by administrative means lack of financial sustainability.

Policy makers have increasingly focused on the role of the market in protecting the environment. Appropriate market instruments can stimulate the private sector's creativity in improving energy efficiency and adopting cleaner processes and technologies, encourage investment in clean production models, and solve complex environmental issues in a more efficient manner. Emissions trading as a market-based environmental economic policy has the characteristics of cost effectiveness and is increasingly used in the environmental management of the United States, the European Union and other countries.

Emissions trading refers to the maximum amount of pollutants allowed by the government environmental department to assess the environmental capacity of a certain area, and then it is broken down into a number of prescribed emission shares, that is, emission rights, based on the total emission control targets. The right to discharge pollutants, like other commodities, is freely traded in the secondary market of emission permits to control the discharge of pollutants. The commonly used transaction method in reality is the emission rights trading conducted among the polluters. For polluters, they can choose between buying pollution rights, self-pollution, and selling pollution rights. Polluters have great flexibility in trading emission rights based on their cost-effectiveness. It can thus be seen that the emissions trading system is a design of a new mechanism for emission rights based on the use of market adjustment methods, and it is also the result of natural selection induced by an increase in the scarcity of environmental capacity. In the discharge and management of pollution, enterprises have also completed the transformation from passive behavior and passive internalization to their own behavioral patterns. Through the formation of the emission trading system, the use of the property rights system effectively encourages manufacturers to take measures to control pollutant emissions in production, relying on profit, price system and property rights to motivate manufacturers to arrange their optimal production plans.

Because the total discharge of pollutants is fixed and the supply curve of pollution rights is completely inelastic, the location of the demand curve determines the price of pollution. The location of the demand curve is determined by the corporate governance costs for pollutants. Those companies that only reduce pollution through high costs will be willing to pay the highest price for the pollution discharge permit. And companies that reduce pollution through low costs have the will to sell their licenses. By selling or auctioning pollution rights and allowing them to be exchanged in the market, property rights can be extended to environmental goods. This expansion helps motivate people to effectively reduce pollution. Therefore, through the emissions trading, it can objectively encourage enterprises to increase technological transformation, increase investment in environmental 
governance, reduce the amount of emissions, and ultimately reduce the demand for emissions rights, to achieve both the purpose of promoting economic growth, but also to protect the environment.

\section{Challenges and Opportunities Faced by the Establishment of a Global Carbon Trading Mechanism}

If the amount of a country's emission reduction is directly or indirectly used by the participating countries of another carbon trading mechanism, the carbon trading mechanism of the two countries can be connected. Theoretically, the linkage between these two mechanisms is economic. Because it increases the scope of emission reduction opportunities, improves market liquidity and optimizes resource allocation. It is also an effective way to prevent vicious competition by allowing bilateral prices to converge in different countries through bilateral connections. In addition, the link between different mechanisms can also reduce price volatility. The carbon trading mechanism is also connected with political considerations. The link makes carbon trading more attractive to countries that do not currently undertake Kyoto emission reduction commitments. The linkage between domestic and regional carbon trading mechanisms will have a chain effect on international consultations on the future international climate mechanism.

However, the international carbon emissions trading mechanism has a basic premise. That is, these countries must establish an environmentally effective carbon trading market. A realistic carbon trading system is not necessarily an effective environment. Therefore, the environmental benefits of acquiring carbon emissions trading mechanism and carbon emissions trading mechanism depend on the design of trading system. Most importantly, the reduction is not achieved from the quota trade, but from total control. Carbon emissions trading reduces the market cost by expanding the scope of emission reduction and participating in emission reduction organizations or enterprises, thereby making emission reduction more effective.

Meanwhile, as a global emissions trading mechanism, its production will certainly bring a lot of challenges. Because it is gradually expanding to the global market through the domestic market and regional markets, it requires a high degree of coordination among the participants. However, there are fundamental differences between the developed countries and the developing countries in the issue of allocation of emission rights. How to coordinate the interests of both parties and adopt a compromise solution acceptable to both parties is very important for the construction of a global trading mechanism. The global carbon emission trading system needs to implement a unified normative clause in the world, which is more difficult. The distribution of benefits between developed and developing countries is the key to determining whether the emissions trading mechanism can operate smoothly.

There is an urgent need for international cooperation to reduce greenhouse gas emissions. Conducting carbon emissions trading is not the fundamental purpose of the L system. The ultimate goal of greenhouse gas emission reduction is to achieve low-cost emission reductions of carbon emissions globally through international carbon emissions trading. This issue has reached consensus in the world. The deadline for emission reduction in the first commitment period of the Kyoto Protocol is coming to an end, and post-Kyoto International's emission reduction provides a new opportunity for the development of a global carbon trading mechanism.

\section{Conclusion}

Global climate change will undoubtedly become an important topic of human society in the twenty-first Century. As a global public property, the atmosphere has decided to recognize and solve the problem of climate change, which requires a wide range of global cooperation from the perspective of protecting the common interests of all mankind. In this process, fairness and efficiency are one of the most significant contradictions, which are specific to the issue of the allocation of responsibility for climate change in developed and developing countries. Developing countries maintain their own development rights with fairness. Developed countries enhance their economic 
interests with efficiency. The international carbon emissions trading mechanism is a means to maintain a balance between fairness and efficiency. On the one hand, the carbon emissions trading mechanism has reduced the cost of greenhouse gas emission reduction in developed countries. On the other hand, the carbon emissions trading mechanism also brings the capital and technology of energy structure and industrial transformation for developing countries. In fact, the creation of such a system mainly stems from the theory of emission trading in environmental economics. This system is characterized by total control and emissions trading.

\section{Acknowledgments}

The paper is supported by the Science and Technology Program of SGCC (Analysis of global energy Internet game behavior supported by big data technology).

\section{References}

[1]. Cong R, Lo a Y. Emission trading and carbon market performance in Shenzhen, China. Applied Energy, 2017, No.193, p. 414-425.

[2]. Liu J P, Zhang X B, Song X H. Regional Carbon Emission Evolution Mechanism and Its Prediction Approach Driven by Carbon Trading - A Case Study of Beijing. Journal of Cleaner Production, 2017, p. 172.

[3]. Liu H P, Song Y, Accounting S O. The Difficulties and Countermeasures of Initiating the National Carbon Emission Trading Market. Economic Review, 2017.

[4]. Shi D, Zhang C, Zhou B, et al. The Implementation Effect and Influencing Factors of Carbon Emission Trading: A Review. Urban \& Environmental Studies, 2017.

[5]. Fan L, Li Z, Wu P. Cement Plant Site Selection Problem with Carbon Emission Trading Mechanism. Proceedings of the Eleventh International Conference on Management Science and Engineering Management. 2018, p. 721-734.

[6]. Mode T, Bellamy S. Asset Classification, Subsequent Measurement and Impairment Testing for Carbon Emission Trading. 2017, p. 65-86.

[7]. Ding H, Min Z. Dynamic Impact of Carbon Emission Trading on China's Macro Economy--Based on CGE Model. International Conference on Education, Management and Computing Technology. 2017.

[8]. Yin Y, Zhou L, Chen Y. Research on China's carbon emission trading market. International Forum on Energy, Environment Science and Materials. 2018. 